\title{
Antenna-on-Package Design: Achieving Near-Isotropic Radiation Pattern and Wide CP Coverage Simultaneously
}

\author{
Zhen Su, Student Member, IEEE, Kirill Klionovski, Hanguang Liao, Yiming Chen, Atef Elsherbeni, \\ Life Fellow, IEEE, and Atif Shamim, Senior Member, IEEE
}

\begin{abstract}
Internet of things (IoT) applications require orientation insensitive wireless devices to maintain stable and reliable communication as well as the localization capability using the global positioning system (GPS). For those reasons, antennas providing near-isotropic radiation pattern with wide circular polarization (CP) coverage are high in demand for IoT applications. In this work, we derive conditions for 3D antenna-on-package (AoP), through a combination of three orthogonal dipoles model and particle swarm optimization (PSO) technique to achieve near isotropic radiation pattern and wide $\mathrm{CP}$ coverage simultaneously. Design guidelines have been provided, and a practical AoP has been designed on a 3D cube package by following this guideline. A prototype has been fabricated and the measured results demonstrate an isotropy of $\sim 93 \%$ and $C P$ coverage of $\sim 18 \%$, which matches well with the predicted results from the theoretical analysis as well as the full wave electromagnetic simulations.
\end{abstract}

Index Terms - circularly polarized antenna; near-isotropic radiation pattern; Orientation insensitive antenna.

\section{INTRODUCTION}

I NTERNET of things (IoT) will bring connectivity to the next level, where billions of smart devices to be designed for making decisions for a large number of applications in an automated fashion. These huge numbers of IoT devices will depend on high speed and uninterrupted wireless communication for their smooth functioning. Thus, a new generation of antennas is required which are space-efficient, low cost and orientation insensitive in terms of stable performance. Low cost and efficient space can be achieved through the antenna-on-package (AoP) concept. The package of the sensors or circuits is used to realize the antennas in addition to providing the required protection from the environment [1-5]. Since the package accommodates the circuits and the battery, etc, typically it is in the shape of a rectangular box, which facilitates the realization of 3D antennas. As the IoT devices can be placed or dispersed arbitrarily, the antenna performance mustn't degrade due to the orientation or position of the package. Thus, it is important to design antennas with near isotropic radiation patterns.

Authors, Z. Su, K. Klionovski, H. Liao, A. Shamim are with the King Abdullah University of Science and Technology (KAUST), Thuwal, 23955-6900, KSA (e-mail: Su.Zhen@kaust.edu.sa, kirill.klionovski@kaust.edu.sa, hanguang.liao@kaust.edu.sa, atif.shamim@kaust.edu.sa). Authors, Y. Chen, A. Elsherbeni are with the Colorado School of Mines, Golden, 80401, USA (Email:yimingchen@mymail.mines.edu_, aelsherb@mines.edu)
Furthermore, for circularly polarized (CP) antennas, the $\mathrm{CP}$ coverage must be enhanced as much as possible.

In literature, many papers report antenna designs with nearisotropic radiation pattern [1-18]. Near isotropic radiation pattern is achieved based on various mechanisms, such as folded dipoles [1-9], split-ring resonators [10], folded slots $[11,12]$ and a combination of electric dipoles with slots or loops [13-16]. However, these papers only focus on optimizing the radiation pattern coverage and not $\mathrm{CP}$ coverage. On the other hand, there are papers focusing only on enhancing the $\mathrm{CP}$ coverage, by bending the dipoles [17-18], double-layer circular patch [19] or with hybrid perturbation method [20], through dielectric resonance [21], by cavity-backed reflector [22] or through conical log spiral $[23,24]$. Although some of them have very large CP coverage [25, 26], their radiation patterns have deep nulls and are thus not suitable for isotropic coverage. There are a handful of papers that have attempted to achieve isotropy in radiation pattern and $\mathrm{CP}$ at the same time. However, their goals for $\mathrm{CP}$ are limited to one direction only, such as boresight and not for wide-angle coverage [27-29]. Also, the figures of merit are not well discussed in these papers, for example, [27-29] do not have the CP coverage information and $[28,29]$ do not have isotropy integral information. Moreover, the reported antennas are planar structures and are thus not suitable for AoP applications. It is worth mentioning here, to the best of our knowledge, that there is no report which investigates conditions for simultaneous isotropy and wide $\mathrm{CP}$ coverage in radiation pattern for 3D AoP designs.

In this paper, we present a three-dipole model, in which the dipoles are orthogonally placed on a 3D package. The excitation phases of the dipoles are optimized through the particle swarm optimization (PSO) algorithm to obtain near isotropic radiation pattern as well as simultaneous enhanced $\mathrm{CP}$ coverage. Design guidelines have been provided, and a design example has been shown based on the guidelines. The prototype has been fabricated and measured to verify the proposed concept. There is a decent agreement between the measured and the predicted results from the derived theoretical model as well as the EM simulations.

\section{THEORETICAL MODEL}

\section{A. Isotropy Definitions}

Isotropy in the radiation pattern is defined differently in the 
literature. The smaller gain variation is, the more near-isotropic antenna is. The antenna can be considered to be isotropic or near-isotropic when the gain variation is smaller than $7 \mathrm{~dB}[3]$. Sometimes a major issue with this method is that the feeding cable can cause a minimum which can affect the overall gain variation and thus foul the value of isotropy. While, in most practical antenna designs, the minimum gain is set as the bottom limit $\mathrm{G}_{\min }$ to support reliable communication. An alternate way is to calculate the integral isotropy factor by finding the ratio of the surface where the gain deviation exceeds the threshold gain level, $T H_{\text {iso }}$, over a complete sphere surrounding the antenna [30]. The sum of the radiation pattern beams solid angles whose gain between the peak gain (PG) and $T H_{\text {iso }}$ below the PG relative to the closed spherical surface surrounding the antenna is a measure of the radiation pattern isotropy. This is reflected mathematically by an integral parameter $I^{t h-i s o}$ as equation (1.a) below.

$$
I^{\text {th-iso }}=100 \% \times \iint_{S_{1}} \frac{\sin \theta d \theta d \varphi}{4 \pi},
$$

The surface $\mathrm{S}_{1}$ is determined by

$$
\mathrm{S}_{1} \text { such that } P G-T H_{\text {iso }} \leq \operatorname{Gain}(\theta, \varphi) \leq P G \text {. }
$$

Similarly, the sum of the radiation pattern beam solid angles that satisfies axial ratio (AR) in the range of $1 \leq A R(\theta, \varphi) \leq$ $\sqrt{2}[31,32]$ relative to closed spherical surface surrounding the antenna is a measure of the radiation pattern CP. A CP coverage factor is introduced as in a normalized integral form $I^{C P}$, to estimate the CP pattern isotropy. This is reflected mathematically by equation (1.b) below.

$$
I^{C P}=100 \% \times \iint_{S_{2}} \frac{\sin \theta d \theta d \varphi}{4 \pi},
$$

The surface $\mathrm{S}_{2}$ is determined by

$$
\mathrm{S}_{2} \text { such that } 1 \leq A R(\theta, \varphi) \leq \sqrt{2} \text {. }
$$

\section{B. The Model of Three Infinitesimal Electric Dipoles}

We have previously shown how to achieve near isotropic AoP design with the help of three orthogonally placed infinitesimal electric dipoles model [5]. Some preliminary work about achieving CP coverage has also been shown in $[31,33]$. In this work, we present the three printed electric dipoles model to achieve simultaneous near isotropy and wide $\mathrm{CP}$ coverage by finding the right phase conditions through PSO. Based on the calculated conditions, a practical antenna design with an appropriate feed network is also conducted in this work (shown in section III).

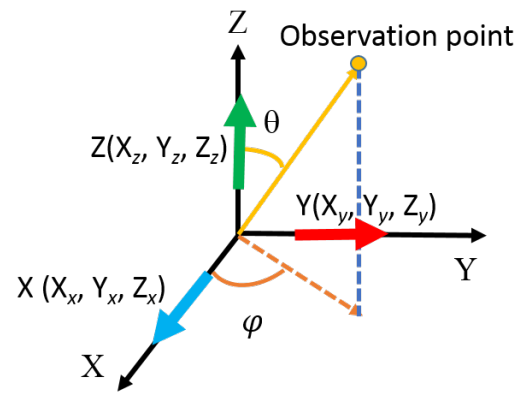

Fig. 1. Configuration of $\mathrm{X}, \mathrm{Y}$ and $\mathrm{Z}$ oriented electrical dipoles.
Fig. 1 shows the three orthogonally placed infinitesimal electric dipoles located arbitrarily in the Cartesian coordinate system (X, Y, Z). The current densities $\mathbf{J}_{\mathbf{x}}, \mathbf{J}_{\mathbf{y}}$ and $\mathbf{J}_{\mathbf{z}}$ of the electrical dipoles oriented along the $\mathrm{X}, \mathrm{Y}$, and $\mathrm{Z}$ axes and located at points $P_{x}\left(\mathrm{X}_{x}, \mathrm{Y}_{x}, \mathrm{Z}_{x}\right), P_{y}\left(\mathrm{X}_{y}, \mathrm{Y}_{y}, \mathrm{Z}_{y}\right)$, and $P_{z}\left(\mathrm{X}_{z}, \mathrm{Y}_{z}\right.$, $\mathrm{Z}_{z}$ ), respectively are given as follows.

$$
\begin{aligned}
& \mathbf{J}_{\mathrm{x}}(\mathrm{X}, \mathrm{Y}, \mathrm{Z})=m_{x} \delta\left(\mathrm{X}-\mathrm{X}_{x}\right) \delta\left(\mathrm{Y}-\mathrm{Y}_{x}\right) \delta\left(\mathrm{Z}-\mathrm{Z}_{x}\right) \mathbf{x}_{0} \\
& \mathbf{J}_{\mathrm{y}}(\mathrm{X}, \mathrm{Y}, \mathrm{Z})=m_{y} \delta\left(\mathrm{X}-\mathrm{X}_{y}\right) \delta\left(\mathrm{Y}-\mathrm{Y}_{y}\right) \delta\left(\mathrm{Z}-\mathrm{Z}_{y}\right) \mathbf{y}_{0} \\
& \mathbf{J}_{z}(\mathrm{X}, \mathrm{Y}, \mathrm{Z})=m_{z} \delta\left(\mathrm{X}-\mathrm{X}_{z}\right) \delta\left(\mathrm{Y}-\mathrm{Y}_{z}\right) \delta\left(\mathrm{Z}-\mathrm{Z}_{z}\right) \mathbf{z}_{0}
\end{aligned}
$$

where $m_{\mathrm{x}}, m_{\mathrm{y}}$ and $m_{\mathrm{z}}$ are the moments of the dipoles along $\mathrm{X}$, $\mathrm{Y}$, and $\mathrm{Z}$ axes, respectively, $\delta($.$) is the Dirac delta-function, \mathbf{x}$, $\mathbf{y}_{0}$ and $\mathbf{z}_{0}$ are the unit vectors along the $\mathrm{X}, \mathrm{Y}$ and $\mathrm{Z}$ axes. The $\mathrm{X}$ oriented dipole has the elevational $E_{\theta}$ and azimuthal $E_{\varphi}$ components of the radiation pattern, as shown in (3).

$$
\begin{gathered}
E_{\theta}(\theta, \varphi)=-\frac{i \eta k m_{x}}{4 \pi} \cos \theta \cos \varphi \mathrm{e}^{i k r_{x}^{\prime} \cos \left(\alpha_{x}(\theta, \varphi)\right)}, \\
E_{\varphi}(\theta, \varphi)=\frac{i \eta k m_{x}}{4 \pi} \sin \varphi \mathrm{e}^{i k k_{x}^{\prime} \cos \left(\alpha_{x}(\theta, \varphi)\right)} .
\end{gathered}
$$

The components of the radiation pattern of the $\mathrm{Y}$ oriented dipole are given in (4).

$$
\begin{gathered}
E_{\theta}(\theta, \varphi)=-\frac{i \eta k m_{y}}{4 \pi} \cos \theta \sin \varphi \mathrm{e}^{i k r_{y}^{\prime} \cos \left(\alpha_{y}(\theta, \varphi)\right)}, \\
E_{\varphi}(\theta, \varphi)=-\frac{i \eta k m_{y}}{4 \pi} \cos \varphi \mathrm{e}^{i k r_{y}^{\prime} \cos \left(\alpha_{y}(\theta, \varphi)\right)} .
\end{gathered}
$$

The radiation pattern of the $\mathrm{Z}$ oriented dipole has only a meridional component as in (5).

$$
E_{\theta}(\theta, \varphi)=\frac{i \eta k m_{z}}{4 \pi} \sin \theta \mathrm{e}^{i k r_{z}^{\prime} \cos \left(\alpha_{z}(\theta, \varphi)\right)} .
$$

Where the phase error coming from the $j$-oriented dipole positions in spherical coordinates is expressed in [31] through spherical components as follows.

$$
\begin{gathered}
r_{j}^{\prime}=\sqrt{X_{j}^{2}+Y_{j}^{2}+Z_{j}^{2}}, \\
\cos \left(\alpha_{j}(\theta, \varphi)\right)=\sin \theta \sin \theta_{j}^{\prime} \cos \left(\varphi-\varphi_{j}^{\prime}\right)+\cos \theta \cos \theta_{j}^{\prime}, \\
\theta_{j}^{\prime}=\arccos \left(\frac{Z_{j}}{r_{j}^{\prime}}\right), \varphi_{j}^{\prime}=\arctan \left(\frac{Y_{j}}{X_{j}}\right) .
\end{gathered}
$$

We assume that the three orthogonal dipoles have equal moments and choose the moment in such a way to normalize the elevational and azimuthal components, i.e. $m_{x}=m_{y}=m_{z}=i 4 \pi /(\eta k)$. The $\mathrm{X}$ and $\mathrm{Y}$ oriented dipoles have a phase shift $\psi_{l}$ between them, while the $\mathrm{X}$ and $\mathrm{Z}$ oriented dipoles have 
a phase shift of $\psi_{2}$ between them. The normalized meridional and azimuthal components of radiation pattern then becomes:

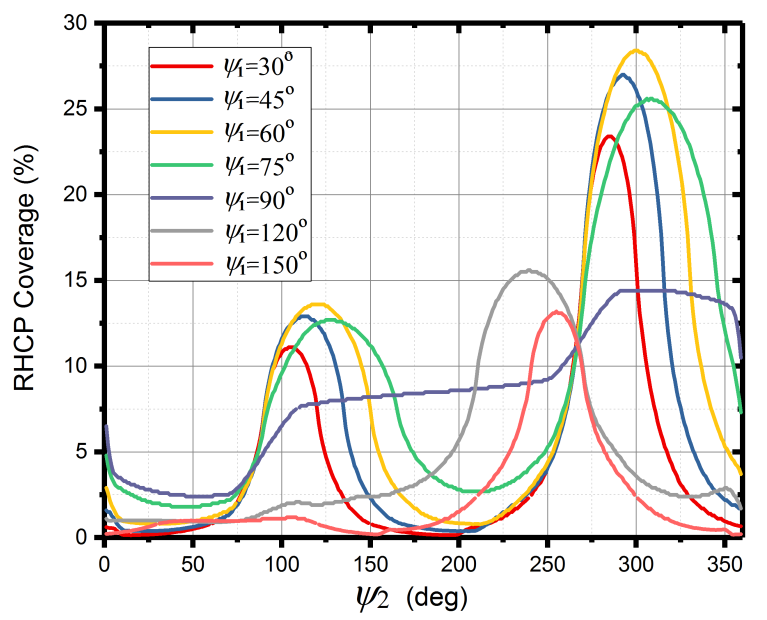

Fig. 3. RHCP far-field zone sphere coverage for the model of three dipoles.

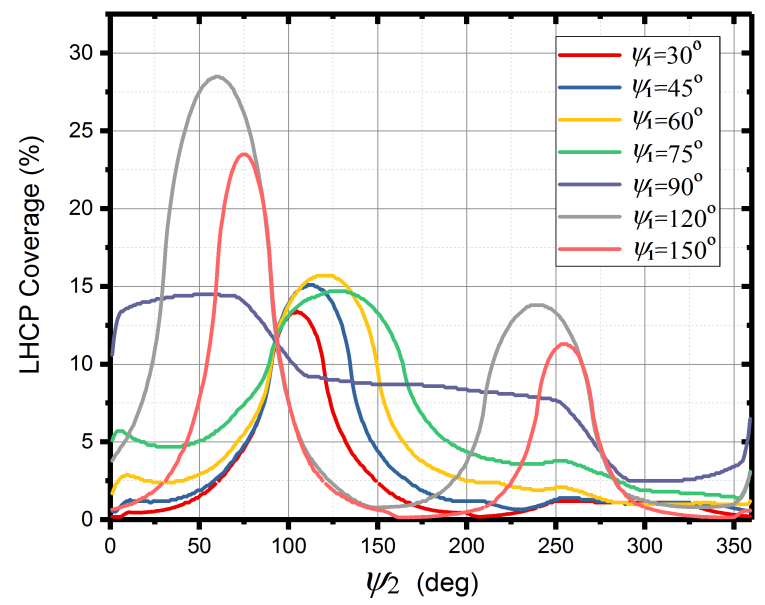

Fig. 4. LHCP far-field zone sphere coverage for the model of three dipoles.

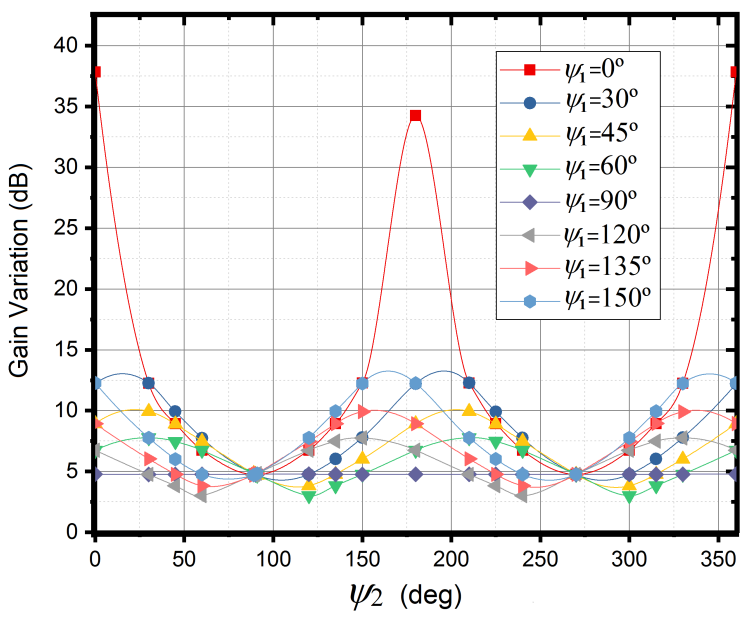

Fig. 5. Isotropy of the model of three dipoles.
$E_{\theta}^{\Sigma}(\theta, \varphi)$

$=\cos \theta \cos \varphi \mathrm{e}^{i k r_{x}^{\prime} \cos \left(\alpha_{x}(\theta, \varphi)\right)}+\cos \theta \sin \varphi \mathrm{e}^{i k r_{y}^{\prime} \cos \left(\alpha_{y}(\theta, \varphi)\right)+i \psi_{1}}$

$-\sin \theta \mathrm{e}^{i k r_{z}^{\prime} \cos \left(\alpha_{z}(\theta, \varphi)\right)+i \psi_{2}}$

$E_{\varphi}^{\Sigma}(\theta, \varphi)=-\sin \varphi \mathrm{e}^{i k r_{x}^{\prime} \cos \left(\alpha_{x}(\theta, \varphi)\right)}+\cos \varphi \mathrm{e}^{i k r_{y}^{\prime} \cos \left(\alpha_{y}(\theta, \varphi)\right)+i \psi_{1}}$.

The CP coverage of the far-field zone, as defined previously in part $\mathrm{A}$, and the power radiation pattern gain variations are calculated through Matlab by manually sweeping the phase difference values $\psi_{1}$ and $\psi_{2}$. The RHCP and LHCP far-field zone sphere coverage and the gain deviation are illustrated in Figs. 3-5. To simplify the problem and reduce the calculation load, the three dipoles are located at the origin for various phase shifts $\psi_{1}$ and $\psi_{2}$. The three-dipole antenna's polarization varies from fully linear to circular polarized. As shown in Fig. 3, the RHCP coverage shows a maximum when $\psi_{2}$ is $300^{\circ}$ and $\psi_{1}$ is equal to $60^{\circ}$. Though, other combinations are also possible such as $\psi_{2}=280^{\circ}$ and $\psi_{1}=45^{\circ}$, however the CP coverage percentage is slightly lower. Similarly, for LHCP, the maximum appears for $\psi_{2}=60^{\circ}$ and $\psi_{1}=120^{\circ}$, as shown in Fig. 4. From Figs. 3 and 4 we can see that a maximum of $28.4 \%$ of CP coverage can be achieved for either RHCP or LHCP. In Fig. 5, the gain variations for various combinations of $\psi_{2}$ and $\psi_{1}$ are moderate (within $\sim 12 \mathrm{dBs}$ ), with the smallest variation of $3 \mathrm{dBs}$ for some combinations such as $\psi_{2}=60^{\circ}$ and $\psi_{1}=120^{\circ}$, or $\psi_{2}=240^{\circ}$ and $\psi_{1}=120^{\circ}$. A Sharp peak in gain variation is seen when $\psi_{1}=0^{\circ}$ and $\psi_{2}=0^{\circ}, 180^{\circ}$ or $360^{\circ}$, as shown in Fig. 5. This happens because the three dipoles are almost in phase. For the best $\mathrm{CP}$ coverage cases (either RHCP or LHCP) and the minimum gain variation of $3 \mathrm{~dB}$, the level of cross-polarization is only $1 \%$. This analysis with the small electric dipoles are a good starting point. However, these dipoles are not practically realizable antenna combination due to the difficulty developing a feeding system that matches the transmission feed line as well as due to dipoles low radiation efficiency. This means that the model of the three small dipoles has to be extended to dipoles with practically realizable lengths. Also, the manual sweep method is not time efficient particularly for the dipoles which have finite lengths and are placed in arbitrary locations.

\section{The Model of Three Half wavelength Dipoles}

As mentioned in the previous section, dipoles with decent finite lengths must be incorporated in the model for practical antenna design. For this work, it is preferred that matching network (stubs, etc.) are not employed, because the metallic lines of the matching network may influence the radiated fields. To simplify the matching problem, half-wavelength dipoles can be utilized here, because theoretically, the real part of the impedance for a half-wavelength dipole is $73 \mathrm{Ohm}$ which is relatively easy to match with the typical 50 -Ohm transmission systems. However, for a half-wavelength dipole, contrary to the small dipole, uniform current distribution assumption can no longer be used, rather a sinusoidal distribution must be incorporated in the model.

Then the current densities for the half-wavelength dipoles along $\mathrm{X}, \mathrm{Y}$ and $\mathrm{Z}$ axes are given by (8). 


$$
\begin{aligned}
& \mathbf{J}_{\mathrm{x}}(\mathrm{X}, \mathrm{Y}, \mathrm{Z})=m_{x} \sin \left[k\left(\frac{L}{2}-\left|\mathrm{X}_{x}\right|\right)\right] \delta\left(\mathrm{Y}-\mathrm{Y}_{x}\right) \delta\left(\mathrm{Z}-\mathrm{Z}_{x}\right) \mathbf{x}_{0} \\
& \mathbf{J}_{\mathrm{y}}(\mathrm{X}, \mathrm{Y}, \mathrm{Z})=m_{y} \delta\left(\mathrm{X}-\mathrm{X}_{y}\right) \sin \left[k\left(\frac{L}{2}-\left|\mathrm{Y}_{y}\right|\right)\right] \delta\left(\mathrm{Z}-\mathrm{Z}_{y}\right) \mathbf{y}_{0} \\
& \mathbf{J}_{z}(\mathrm{X}, \mathrm{Y}, \mathrm{Z})=m_{z} \delta\left(\mathrm{X}-\mathrm{X}_{z}\right) \delta\left(\mathrm{Y}-\mathrm{Y}_{z}\right) \sin \left[k\left(\frac{L}{2}-\left|\mathrm{Z}_{z}\right|\right)\right] \mathbf{z}_{0}
\end{aligned}
$$

where $L$ is the total length of the half-wavelength dipole. For the half-wavelength dipoles placed along the three axes in the cartesian coordinate system, the normalized fields are given in (9).

$$
\begin{gathered}
E_{x \theta}(\theta, \varphi)=\frac{2 \cos \left(\frac{k L}{2} \sin \theta \cos \phi\right)}{1-\sin ^{2} \theta \cos ^{2} \phi} \cos \theta \cos \varphi \mathrm{e}^{i k r_{x}^{\prime} \cos \left(\alpha_{x}(\theta, \varphi)\right)}, \\
E_{x \varphi}(\theta, \varphi)=-\frac{2 \cos \left(\frac{k L}{2} \sin \theta \cos \phi\right)}{1-\sin ^{2} \theta \cos ^{2} \phi} \sin \varphi \mathrm{e}^{i k r_{x}^{\prime} \cos \left(\alpha_{x}(\theta, \varphi)\right)} . \\
E_{y \theta}(\theta, \varphi)=\frac{2 \cos \left(\frac{k L}{2} \sin \theta \sin \phi\right)}{1-\sin ^{2} \theta \sin ^{2} \phi} \cos \theta \sin \varphi \mathrm{e}^{i k r_{y}^{\prime} \cos \left(\alpha_{y}(\theta, \varphi)\right)}, \\
E_{y \varphi}(\theta, \varphi)=\frac{2 \cos \left(\frac{k L}{2} \sin \theta \sin \phi\right)}{1-\sin ^{2} \theta \sin { }^{2} \phi} \cos \varphi \mathrm{e}^{i k r_{y}^{\prime} \cos \left(\alpha_{y}(\theta, \varphi)\right)} . \\
E_{z \theta}(\theta, \varphi)=-\frac{\cos \left[\frac{\pi}{2} \cos \theta\right]}{\sin \theta} \mathrm{e}^{i k r_{z}^{\prime} \cos \left(\alpha_{z}(\theta, \varphi)\right)} .
\end{gathered}
$$

Finding the right phase conditions for the half-wavelength dipoles is not easy by manually sweeping the phase differences between the dipoles, thus an automated optimization routine is required for this purpose.

\section{Particle Swarm Optimization (PSO)}

Particle swarm optimization (PSO) is a population-based evolutionary optimization algorithm [34]. This optimization method employs a large number of swarm members to record, share, and compare the individual solutions and then iteratively improve the candidate solution. It can achieve the optimization target for a relatively smaller number of optimization parameters and avoids stagnation at the local optimum point without requiring an initial estimation at the start. The PSO flowchart, pertinent to this work, is shown in Fig. 6. The algorithm begins by defining the parameters, the cost function, and the optimization boundary. The three parameters, isotropy threshold $\mathrm{TH}_{\text {iso, }}$, its near isotropy coverage limit $\mathrm{I}^{\text {th-iso }}$, and the minimum gain $G_{\min }$, are set by designers for their specific environments and requirements. Other than those three customized parameters, the excitation phases of the three dipoles are defined as the particle positions. Another two important vectors Pbest and Gbest are defined to store the intermediate information to generate the new velocity of the particles for the next movements. The former keeps the personal best position and lowest cost value of every single particle, and the latter stores the global best position and lowest cost among all the particles. The velocity of each particle movement is defined as the particle movement step, proportional to the difference between its position and the global best position. The objective cost function $\mathrm{F}$ is defined as the reverse function of the $\mathrm{CP}$ coverage determined by the antenna radiation model. Then those parameters for a swarm of particles are initialized. The initial positions of all the particles are randomly selected, and the initial velocity is zero. The two vectors, Pbest and Gbest, are set to have empty position values and infinite large cost values. In the next step, a time-domain iteration nested with a space domain iteration is performed. The first particle position and cost function are filled into its Pbest as the space domain iteration begins. The first stop criterion is checked whether the antenna is near-isotropic or not, depending on the isotropy requirement set up by the user. The near isotropic antenna standard is characterized by the $G_{\min }$, Thiso, and $\mathrm{I}^{\text {th-iso }}$. $\mathrm{I}^{\text {Pth-iso }}$ is the temporary value that represents the particle isotropy integral parameter $\mathrm{I}^{\text {th-iso }}$, and similarly, Gp is the temporary vector which records the minimum gain across the whole sphere. If $\mathrm{I}^{\text {Pth-iso }}>\mathrm{I}^{\text {th-iso }}$, and $\mathrm{GP}_{\mathrm{P}}>\mathrm{G}_{\mathrm{min}}$, the radiation pattern is near-isotropic. Otherwise, another space domain iteration is triggered with Particle Index increasing by 1 . Once the condition of near isotropy is met then it moves forwards to the next step where the antenna CP coverage is calculated. After that Pbest and Gbest are updated accordingly. Once the space domain iterations are completed, meaning that all the particles' current positions and cost values are calculated, the program runs to the next time-domain iteration. When the number of time-domain iterations reach the set limit, the required excitation phase conditions for 3 dipoles model and the corresponding $\mathrm{CP}$ and isotropy coverage are obtained. In Matlab, the computation time could be further reduced by a parallel computation that realizes the space iteration through matrix operation.

For practical antenna design, the three half-wavelength dipoles may be placed away from each other to minimize the coupling between them, as shown in Fig. 7. This is a complicated optimization problem as compared to the small dipoles at the origin because the antennas are electrically large and have been strategically placed on the package in an orthogonal fashion. As can be seen in Figs. 3 and 4, multiple local peaks may appear in the CP coverage optimization, thus, PSO is a good option for this problem because it can easily reach the global maximum to provide phase conditions for simultaneously achieving near isotropy and wide CP coverage.

As shown in Fig. 7, the dipoles are placed on the three orthogonal edges of the cubic package with air as the medium. The parameters $W_{d}$ and $L_{d}$ represent the width and total length of the dipoles, respectively. The centers of the half-wavelength dipoles are located at $(\mathrm{W} / 2,0,0),(\mathrm{W}, \mathrm{L} / 2, \mathrm{H})$ and $(0, \mathrm{~L}, \mathrm{H} / 2)$ along the $\mathrm{X}, \mathrm{Y}$ and $\mathrm{Z}$, respectively. The target of the PSO algorithm is to figure out the phase conditions to maximize the CP coverage, while maintaining the radiation pattern isotropy higher than isotropy limit $I^{\text {th-iso }}$, as defined in (0.a). The optimization procedure minimizes a defined cost function that 


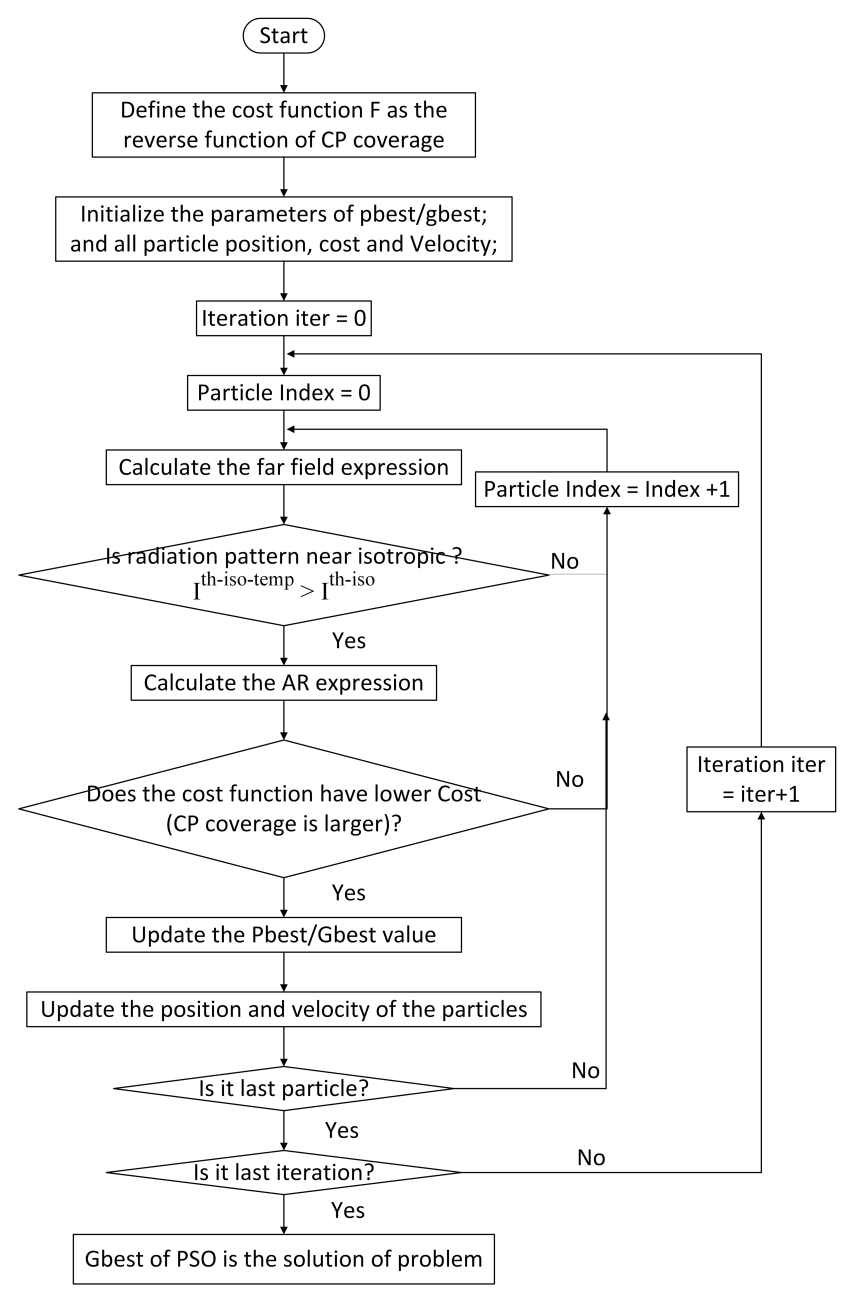

Fig: 6. PSO flowchart. * $\mathrm{I}^{\text {Pth-iso }}>\mathrm{I}^{\text {th-iso }}$, and $\mathrm{GP}_{\mathrm{P}}>\mathrm{G}_{\mathrm{min}}$.

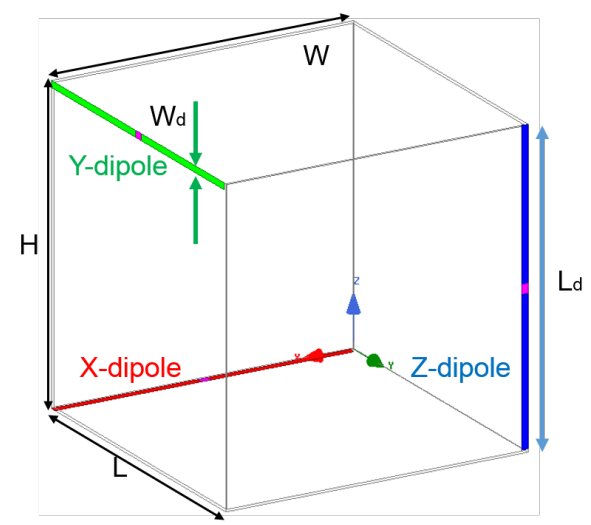

Fig. 7. Configuration of the three off-origin half wavelength dipoles on the package. The parameters $\mathrm{L}=\mathrm{W}=\mathrm{H}=95 \mathrm{~mm}$ are the dimensions of the cubic package for antenna at $1.57 \mathrm{GHz}$ operating frequency.

is a reverse function of the $\mathrm{CP}$ coverage factor in $(0 . \mathrm{b})$ as given by $(10)$.

$$
F=\frac{1}{I^{C P}},
$$

The solution is searched within the search-space boundary

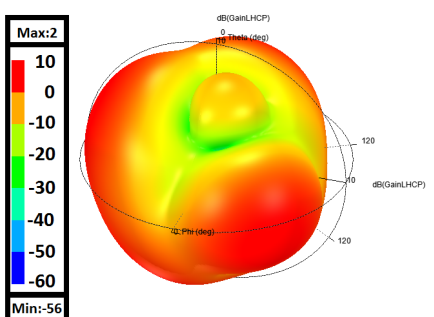

(a)
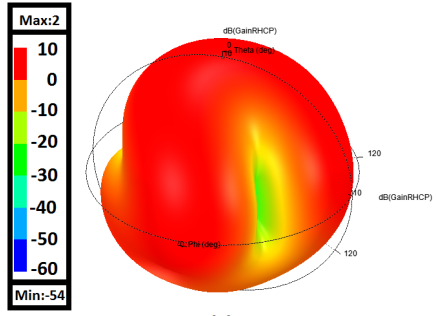

(c)

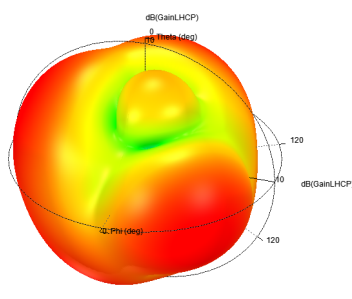

(b)

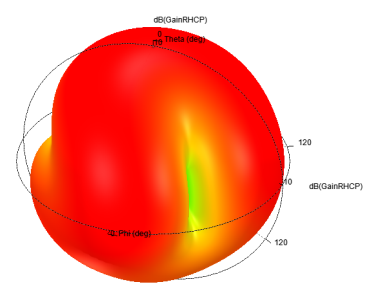

(d)
Fig: 8. LHCP gain of (a) Theoritical model with PSO optimization and (b) HFSS simulation model. RHCP gain of (c) Theoritical model with PSO optimization and (d) HFSS simulation model

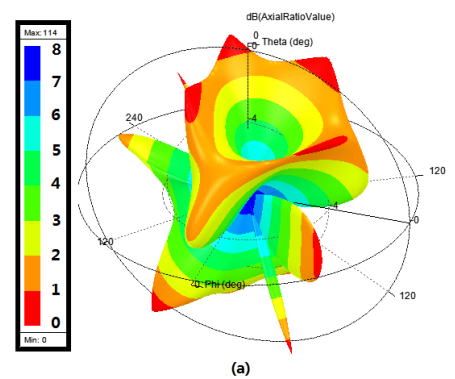

(a)

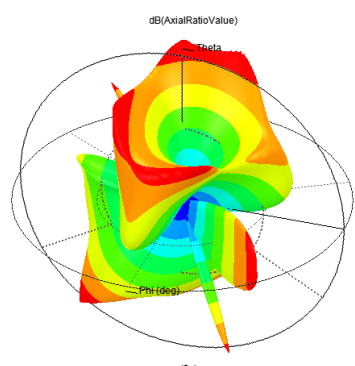

(b)
Fig: 9. The CP coverage for three half wavelength dipoles (a) Theoritical model with PSO optimization (b) HFSS simulation model

for good near isotropy that satisfies $I^{\text {Pth-iso }}>I^{\text {th-iso }}$, and Gp $>$ $\mathrm{G}_{\mathrm{min}}$. For example, we set $T H_{i s o}=7 \mathrm{~dB}$ and $I^{t h-i s o}=90 \%, \mathrm{G}_{\min }$ $=-10 \mathrm{dBs}$. The optimized phase difference between the $\mathrm{X}$ and $\mathrm{Y}$ dipoles is $117^{\circ}$, whereas the phase difference between the $\mathrm{X}$ and $\mathrm{Z}$ dipoles is $236^{\circ}$, with all 3 ports fed with equal power level. The theoretical model in section $\mathrm{C}$ calculated by Matlab predicts a $98 \%$ isotropy and $21.8 \%$ CP coverage. For verification, the optimized phase difference values from the PSO have been applied to a model in Fig.7 operating at $1.57 \mathrm{GHz}$ utilized in the full-wave EM simulator, Ansys HFSS. The EM simulation results are close to the predicted values from the theoretical model as can be seen from Figs. 8 and 9, except for some very minor differences. This comparison is a good validation of the theoretical model. The $3 \mathrm{D}$ package has been kept hollow from inside to accommodate the driving electronics, which matches the concept of AoP. Though not shown here, the antenna has been simulated with a metal cube (representative of the driving electronics, for example, a PCB with a typical dimension around $\frac{\lambda}{8} * \frac{\lambda}{8} * \frac{\lambda}{8}$ placed either at the corner or the center of the package, $\lambda$ is the wavelength of the operation frequency) and the performance of the AoP remains almost stable (around 22\% CP coverage). Having said that, if a larger metallic structure such as a battery, etc. needs to be 
placed inside the package then the antenna may need to be optimized a bit, as we have shown in our previous works [3-5].

\section{E. Design Guidelines}

To make the design process systematic, a design guideline containing basic steps to design a near-isotropic antenna with wide CP polarization simultaneously is given as follows.

First, get the total field expressions of the dipoles groups following the steps:

1. Choose any corner of the package as the Cartesian coordinate system origin. The choice of the origin position does not make any difference to the final results.

2. Locate the three half-wavelength dipoles along with the $\mathrm{X}, \mathrm{Y}$, and $\mathrm{Z}$ directions respectively on the surface of the package. Note down the center positions of the 3 dipoles referred to the origin as $r_{j}^{\prime}(\mathrm{j}=\mathrm{x}, \mathrm{y}$, or $\mathrm{z})$ in Eq. (6.a).

3. Use the dipole center positions in Eq. (6.a-6.c) to calculate the spatial phase errors $\left({ }^{k r_{j}^{\prime}} \cos \left(\alpha_{j}(\theta, \varphi)\right)\right.$ ).

4. Derive the total E-field expressions for the three dipoles through Eq. (9.a-9.e) using the spatial phase errors calculated in step 3.

Second, drive the excitation phase conditions for each dipole with PSO techniques for the user's requirements. There are four steps to accomplish this procedure as below.

5. The isotropy threshold $T H_{\text {iso }}$, its near isotropy coverage limit $I^{\text {th-iso }}$, and the minimum gain $\mathrm{G}_{\min }$ can be defined by the readers for their requirements. In our example as mentioned, we choose $T H_{\text {iso }}=7 \mathrm{~dB}$ as isotropy threshold, coverage limit $I^{t h-i s o}=90 \%$, and the $\mathrm{G}_{\min }=-10 \mathrm{~dB}$.

6. At this point, an optimization method can be used. We have used the PSO technique and the algorithm flow that has been provided in the flow chart (Fig. 6). Utilize the expressions derived in step 4, along with the reader-defined isotropy threshold $T H_{\text {iso }}$, near isotropy coverage limit $I^{\text {th-iso }}$, and minimum gain $\mathrm{G}_{\min }$ from step 5 in the first decision box in Fig. 6 .

7. Run the PSO code to get the excitation phase conditions for three half-wavelength dipoles which lead to the maximum $\mathrm{CP}$ coverage under the condition defined by the readers.

8. Set the excitation phases of the dipoles in the HFSS model using PSO optimization results. Verify the radiation property performance calculated in the PSO with HFSS simulation results.

Third, use the phase conditions derived from the theoretical model to a practical design and the detailed steps are list below.
9. Design a feeding network that ensures the required phases (calculated in step 7) reach the dipoles with equal magnitudes.

10. Final optimization can now be done for the complete AoP (dipoles with feeding network) in an EM simulator. Adjust the feeding network to optimize the complete system performance, if needed.

\section{Practical Antenna Design}

In this section, a practical AoP design example is provided based on the design guideline provided in the last section to prove the feasibility of our design guidelines.

To simplify the design process and get a better match with the previous simulation, the package dimensions are set to be $\mathrm{L}=\mathrm{W}=\mathrm{H}=95 \mathrm{~mm}$, which is slightly longer than a halfwavelength dipole. Low-loss Rogers ${ }^{\circledR}$ laminates with a dielectric constant $\varepsilon_{\mathrm{r}}=3.3$, loss tangent $\tan \delta=0.0015$ and thickness $\mathrm{t}=0.762 \mathrm{~mm}$ have been used for the package fabrications. Following the first step, the origin is selected as the same as the one shown in the PSO example (Fig. 7). While it could be changed to another corner and it does not affect the final results. Following step 2, the three half-wavelength dipoles, $\mathrm{X}, \mathrm{Y}$, and $\mathrm{Z}$, are located along the three edges of the package as shown in Fig. 7, and their centers are noted down as $(47.5 \mathrm{~mm}, 0,0),(95 \mathrm{~mm}, 47.5 \mathrm{~mm}, 95 \mathrm{~mm})$ and $(0,95 \mathrm{~mm}$, $47.5 \mathrm{~mm}$ ), respectively. Then, following step 3 , the spatial error of each dipole is calculated by Eq.(6.a-6.c), and the total E-field is expressed using the Eq.(9.a-9.e) with the spatial errors calculated from step 3.

In step 5, the near isotropy requirements are set up by the user for their requirements, and here in our design example, we have set $T H_{i s o}=7 \mathrm{~dB}$ and $I^{t h-i s o}=90 \%$ to have a better match with the example we showed in the last section. Next, following step 6, the PSO codes are updated with the parameters set up in step 4 and 5. The readers can apply their optimization programs for the calculation, and here we choose the PSO algorithm shown in Fig. 6 for the phase conditions calculations. Following step 7 , the PSO code is executed to get the excitation phase conditions for minimum cost function, which means the optimized CP coverage is obtained. Here in our example, the phase conditions are $\psi_{1}=117^{\circ}$ and $\psi_{2}=236^{\circ}$ with an overall CP coverage of $21.8 \%$. In step 8 , the obtained phase conditions are implemented in the HFSS model. For our example, the simulation results in HFSS match the theoretical model prediction as shown in Fig. 8 and 9.

After getting a good match between the theoretical model and HFSS simulations, the next phase is to design a practical AoP with the appropriate feeding network to feed the three orthogonal dipoles. As shown in step 8, each dipole is fed through an independent lumped port in HFSS simulation. In step 9 , a feeding network which provides the phase conditions $\left(\psi_{1}=117^{\circ}\right.$ and $\left.\psi_{2}=236^{\circ}\right)$ is required to realize the final AoP. It is difficult to realize this feed arrangement for practical antenna design as this requires either an individual excitation cable or an individual driving circuit for each dipole. Other than 


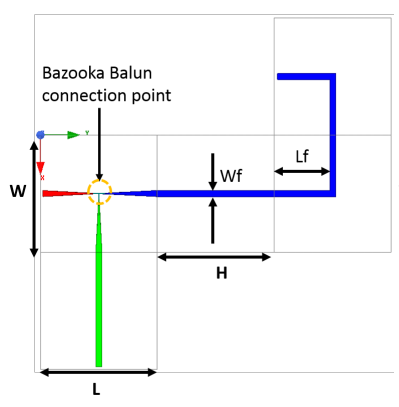

(a)

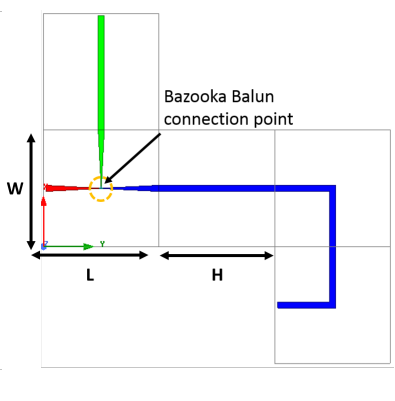

(b)
Fig: 10. The planar phase shifter (a) top view. (b)bottom view

the practical use, for testability as well, three half-wavelength dipoles fed by a single SMA connector is less complex and more convenient than three coaxial lines with three baluns to feed the dipoles individually. Thus, for practical antenna design, it is better to utilize a single driving network. A balun is also required to transit single-ended signal from coaxial cable to differential to feed dipole antennas. In step 9, to feed the three orthogonal dipoles on the cubic package with the correct phases, a phase shifter with three parallel output lines has been designed, as shown in Fig. 10. Though the feed network has to be implemented on the 3D package, it is initially designed on a planar surface such that when folded, all the squares shown in Fig. 10 become various faces of the cubic package. Each square in Fig. 9 has the same dimensions $95 \mathrm{~mm}$ as that of an individual side of the package. To feed the differential antennas, parallel lines have been designed such that one part of the line is on the top side of the substrate, whereas the other part is on the bottom side as shown in Fig.10. This arrangement also helps in minimizing the effect of these lines on dipoles' radiation. The parallel line is designed to be as short as possible to further reduce the effects on the radiating field. In Fig. 10, the red parallel line feeds the $\mathrm{X}$-axis dipole, the green one feeds the $\mathrm{Y}$ axis dipole and the blue one feeds the Z-axis dipole. The width of the parallel lines determines their characteristic impedance and their lengths determine the output port phases. The difference in lengths between the parallel lines provides the phase differences between the output ports, which are the key factors affecting the antenna performance. The tapered part of the parallel lines helps in impedance matching with the dipoles. Once the right phases, according to the theoretical method, have been achieved, the individual substrates are folded to form the

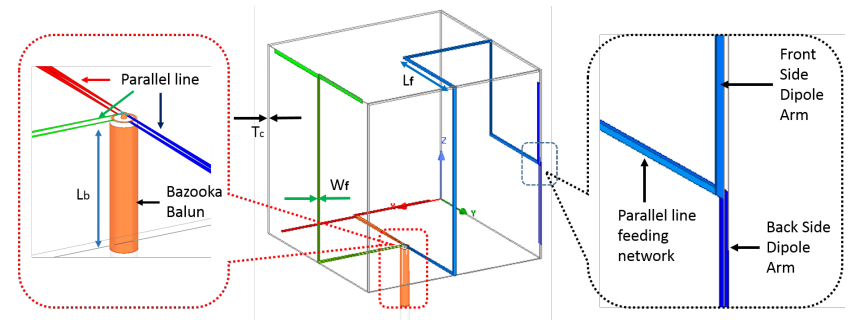

Fig: 11. Three half wavelength dipoles with feeding network. Antenna width $=\mathrm{Wd}=2 \mathrm{~mm}$, Anetnna length $=\mathrm{Ld}=82 \mathrm{~mm}$, feeding lines width $=\mathrm{Wf}=1.2 \mathrm{~mm}$, TaperedSection Width $=$ from $1.2 \mathrm{~mm}$ to $0.3 \mathrm{~mm}$, Additional phase shift length for $\mathrm{Z}$ axis dipole $=\mathrm{Lf}=47 \mathrm{~mm}$

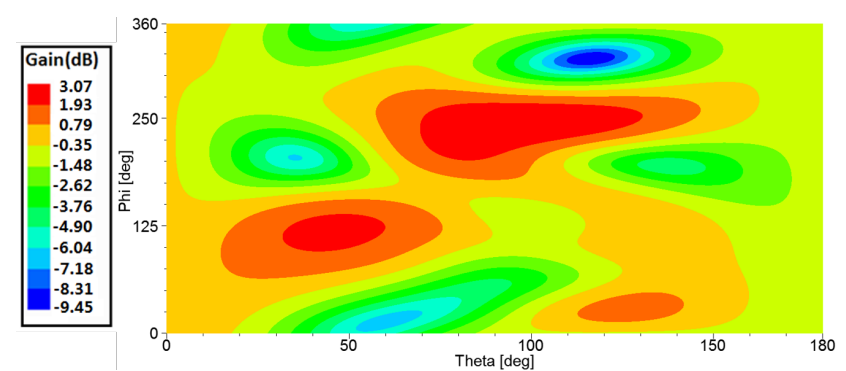

Fig: 12. The simulated antenna radiation pattern in contour plot

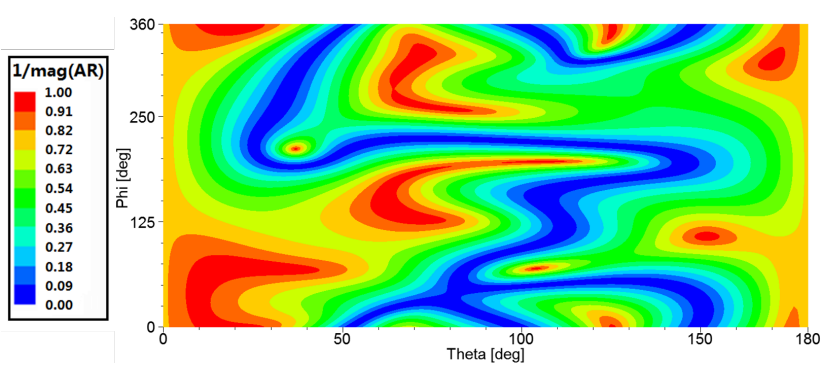

Fig. 13. antenna CP coverage at target frequency

cubic package. Simulation results confirm that the folding of the feed network has a negligible effect on its planned performance. The feed network connects to a Bazooka balun at the intersection point of the three parallel lines to transform the differential signals to the single-ended mode for the coaxial cable, as shown in Fig. 11. The length of the Bazooka balun is designed to be a quarter wavelength long at $1.57 \mathrm{GHz}$, which is $l_{b}=28.9 \mathrm{~mm}$.

After step 9, the three dipoles connected with the feeding network are shown in Fig. 11, where the two dipole's arms are placed on either side of the substrate, consistent with the parallel line layout mentioned above. Despite the precautions are taken with the feed network, the finite width and lines of the feedline do affect the antenna radiation and thus the CP coverage of the antenna with the feeding network is not as good as has been predicted from the theoretical model and initial simulations (without the feed network). Therefore, in step 10, the dimensions of the antennas and phase shifters are optimized slightly to get good CP coverage. The re-optimized phase difference between the $\mathrm{X}$ and $\mathrm{Y}$ dipoles is $88^{\circ}$, whereas the phase difference between the $\mathrm{X}$ and $\mathrm{Z}$ dipoles are $276^{\circ}$. The final dimensions for the antenna and the feed network are given in the caption of Fig. 11.

The simulated results for the radiation pattern as well as $\mathrm{CP}$ coverage are shown in Figs. 12 and 13. It can be calculated from the simulation result that a $7 \mathrm{~dB}$ near isotropy coverage of $92 \%$ is achieved which is somewhat lower from the initial prediction of $98 \%$ but still acceptable given that the initial prediction did not include the effects of the feeding network. Further, a CP coverage of $21 \%$ is achieved which is marginally below the initial prediction of $21.8 \%$. The antenna has a gain of $3 \mathrm{dBi}$ at $1.575 \mathrm{GHz}$.

\section{FABRiCATION AND MEASUREMENTS}

The antenna prototype has been created by fabricating six individual faces of the cube with the required metallization and 


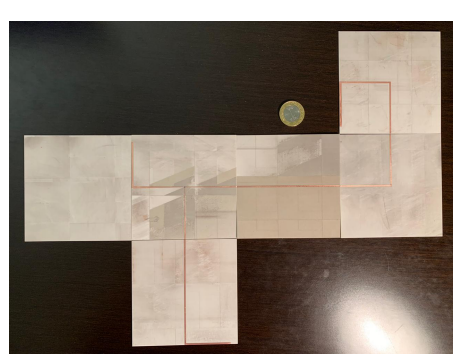

(a)

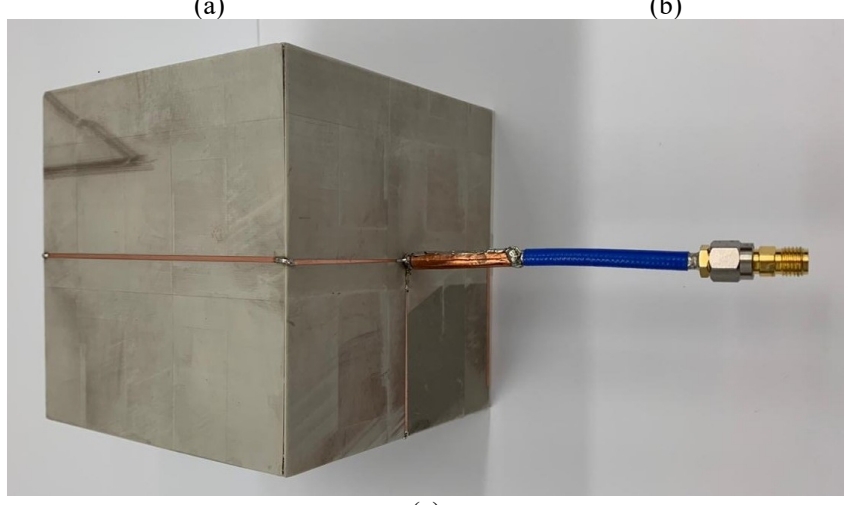

(c)

Fig. 14. (a). Fabricated six roger board with metallic patterns for antenna and phase shifter. (b). The glued antenna on package (c).The antenna on package with bazooka balun

then assembling them in the form of a cube, as shown in Fig. 14. Each board is fabricated individually by LPKF using the low-loss Rogers ${ }^{\circledR}$ boards, as shown in Fig. 14. (a). The metal traces on the boards are soldered at appropriate junction points to strengthen the metal connection. The bazooka balun is fabricated by realizing the sleeve through the copper tape on the coaxial line. The balun is then soldered to the parallel traces on the bottom plate. Finally, all the faces of the cube are attached with the help of super glue to form the cube. The complete prototype is shown in Fig. 14 (b) and (c).

The input reflection coefficient of the antenna has been measured through Agilent ${ }^{\circledR}$ VNA Keysight N9912A and the results are shown in Fig. 15. As can be seen from the measurement results, the antenna is matched to $50 \mathrm{Ohms}$ from $1.34 \mathrm{GHz}$ to $1.81 \mathrm{GHz}$, which is quite similar to the simulated bandwidth. Overall, there is a good match between the

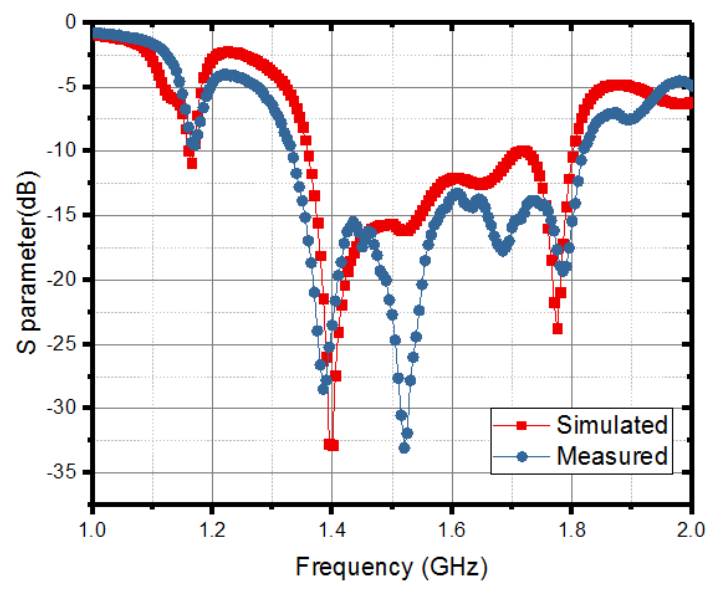

Fig. 15. Measured and simulated $\mathrm{S}$ parameters of the total antenna system.

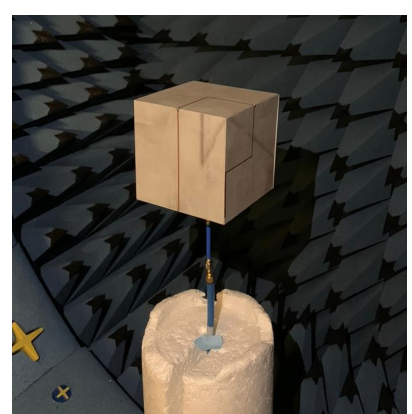

(a)

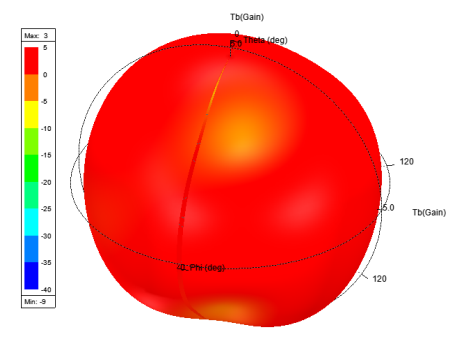

(b)
Fig. 16. (a). The radiation pattern measurement setup in Satimo Chamber (b). the $3 \mathrm{D}$ radiation pattern.

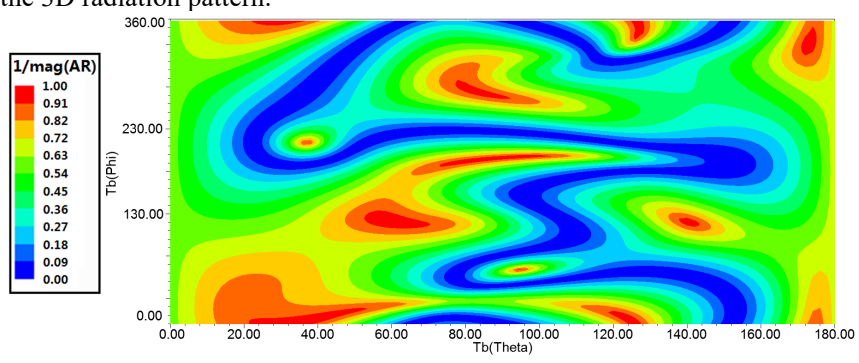

Fig. 17. Measured CP coverage.

simulation and measurement results. However, there is a slight shift in the measured $\mathrm{S}_{11}$ curve (approximately $50 \mathrm{MHz}$ ) around the center frequency, which could be due to the fabrication tolerances. The other difference is that it is very well-matched resonance at $1.52 \mathrm{GHz}$, which does exist but is not that prominent in the simulations. It is worth mentioning here that the narrowest parts of the parallel strip line are $0.3 \mathrm{~mm}$ and they have been connected to the bazooka balun through soldering which may have introduced some additional differences. Table I. Comparison between simulated and measured results

\begin{tabular}{c|c|c}
\hline & $7 \mathrm{~dB}$ isotropy & CP coverage \\
\hline Simulation & $94.01 \%$ & $17.3 \%$ \\
\hline Measurement & $92.86 \%$ & $17.2 \%$ \\
\hline
\end{tabular}

Moreover, the bazooka balun has been manually fabricated and it appears that the shorted stub length is not as accurate as has been simulated.

To obtain the radiation parameters, the proposed antenna is measured in Satimo ${ }^{\circledR}$ anechoic chamber, as shown in Fig. 16. (a). The best isotropy and CP coverage performance is observed at $1.52 \mathrm{GHz}$ instead of the center frequency of $1.57 \mathrm{GHz}$. This $50 \mathrm{MHz}$ shift is consistent with the impedance measurements, as described above. The measured $3 \mathrm{D}$ radiation pattern at 1.52 $\mathrm{GHz}$ is shown in Fig. 16. (b). The measured $7 \mathrm{~dB}$ isotropy at $1.52 \mathrm{GHz}$ is $92.86 \%$, which is quite close to the simulation results, as can be seen in Table I. The antenna's measured CP coverage is shown in Fig. 17, with a CP coverage of $17.2 \%$ at $1.52 \mathrm{GHz}$. This value is also quite close to the simulated value at $1.52 \mathrm{GHz}$, however, it is somewhat lower than the $21 \% \mathrm{CP}$ coverage predicted by simulations at $1.57 \mathrm{GHz}$. As explained above, this may be due to the $50 \mathrm{MHz}$ frequency shift observed in measurements. Nonetheless, in simulations, the performance is quite similar at $1.52 \mathrm{GHz}$, which validates the proposed theory and design concept. 
It is well known that antennas' radiation performance gets affected by nearby objects and the proposed antenna is not exempted from that. However, if the antenna system is smart enough to sense the environment, then the phases of the signals fed to the individual elements can be adjusted to reconfigure the radiation pattern accordingly.

\section{CONCLUSION}

A combination of three orthogonal dipoles' model and PSO technique has been utilized in this work to design 3D AoP with near isotropic radiation pattern and wide circular polarization coverage. Appropriate phase conditions to achieve maximum $\mathrm{CP}$ coverage and decent isotropy from the theoretical model are then utilized to demonstrate a practical AoP. A design guideline is provided for readers to design AoP which can achieve maximum $\mathrm{CP}$ and isotropy coverage simultaneously. A design example has been presented based on the design guidelines. The prototype shows a decent performance and matches well with the predicted performance from the theoretical analysis. The methodology presented in this work can be utilized to design and optimize antennas on 3D packages for future IoT applications. Also, the radiating structure can be replaced by other suitable antenna types and thus more advanced functions, such as different orbital angular momentum beams, can be realized [35].

\section{REFERENCES}

[1] C. M. Kruesi, R. J. Vyas, and M. M. Tentzeris, "Design and development of a novel 3-D cubic antenna for wireless sensor networks (WSNs) and RFID applications," IEEE Transactions on Antennas and Propagation, vol. 57, no. 10, pp. 3293-3299, 2009.

[2] I. T. Nassar, and T. M. Weller, "Development of novel 3-D cube antennas for compact wireless sensor nodes," IEEE Transactions on Antennas and Propagation, vol. 60, no. 2, pp. 1059-1065, 2012.

[3] M. F. Farooqui, C. Claudel, and A. Shamim, "An inkjet-printed buoyant 3-D Lagrangian sensor for real-time flood monitoring," IEEE Transactions on Antennas and Propagation, vol. 62, no. 6, pp. 3354-3359, 2014.

[4] M. F. Farooqui, M. A. Karimi, K. N. Salama, and A. Shamim, "3D Printed Disposable Wireless Sensors with Integrated Microelectronics for Large Area Environmental Monitoring, " Advanced Materials Technologies, 2017.

[5] Z. Su, K. Klionovski, R. M. Bilal, and A. Shamim, "A Dual Band Additively Manufactured 3-D Antenna on Package With Near-Isotropic Radiation Pattern," IEEE Trans. Antennas Propag., vol. 66, no. 7, pp. 3295-3305, July 2018.

[6] C. Cho, H. Choo, and I. Park, "Printed symmetric inverted - F antenna with a quasi - isotropic radiation pattern," Microwave and Optical Technology Letters, vol. 50, no. 4, pp. 927-930, 2008.

[7] H.-K. Ryu, G. Jung, D.-K. Ju, S. Lim, and J.-M. Woo, "An electrically small spherical UHF RFID tag antenna with quasi-isotropic patterns for wireless sensor networks," IEEE Antennas and Wireless Propagation Letters, vol. 9, pp. 60-62, 2010.

[8] G. Pan, Y. Li, Z. Zhang, and Z. Feng, "Isotropic radiation from a compact planar antenna using two crossed dipoles," IEEE Antennas and Wireless Propagation Letters, vol. 11, pp. 1338-1341, 2012.

[9] C. Deng, Y. Li, Z. Zhang, and Z. Feng, "A wideband isotropic radiated planar antenna using sequential rotated L-shaped monopoles," IEEE Transactions on Antennas and Propagation, vol. 62, no. 3, pp. 1461-1464, 2014.

[10] J.-H. Kim, and S. Nam, "A compact quasi-isotropic antenna based on folded split-ring resonators," IEEE Antennas and Wireless Propagation Letters, vol. 16, pp. 294-297, 2017.
[11] C. Deng, Y. Li, Z. Zhang, and Z. Feng, "Design of a three-dimensional folded slot antenna with quasi-isotropic radiation pattern." Proc. IEEE Int. Symp. Antennas Propag. USNC/URSI Nat. Radio Sci. Meeting, pp. 588589,2015 .

[12] S. Shamsinejad, F. De Flaviis, and P. Mousavi, "Microstrip-Fed 3-D Folded Slot Antenna on Cubic Structure," IEEE Antennas and Wireless Propagation Letters, vol. 15, pp. 1081-1084, 2016.

[13] S. Long, "A combination of linear and slot antennas for quasi-isotropic coverage," IEEE Transactions on antennas and propagation, vol. 23, no. 4, pp. 572-576, 1975.

[14] C. Cho, H. Choo, and I. Park, "Broadband RFID tag antenna with quasiisotropic radiation pattern," Electronics Letters, vol. 41, no. 20, pp. 1091$1092,2005$.

[15] L. Liang, and S. V. Hum, "A low-profile antenna with quasi-isotropic pattern for UHF RFID applications," IEEE Antennas and Wireless Propagation Letters, vol. 12, pp. 210-213, 2013.

[16] C.-H. Wu, and T.-G. Ma, "Miniaturized self-oscillating active integrated antenna with quasi-isotropic radiation," IEEE transactions on Antennas and Propagation, vol. 62, no. 2, pp. 933-936, 2014.

[17] Wang, S.W. Cheung, W.Y. Wang, M. Li, T.I. Yuk, "A broadband and wide beamwidth dual circularly polarized antenna using crossed bent dipoles", 2017 11th European Conference on Antennas And Propagation (Eucap), pp. 1936-1939, 2017

[18] X.-Y. He, L. Chang, and L.-L. Chen, "A multifunction broad-beam antenna with dual bands and dual circular-polarizations.", 2016 IEEE MTT-S International Wireless Symposium (IWS), pp. 1-4, 2016

[19] Y. Jie, L. Chun-lan, H. Yong-jin, J. Jian-xin, and J. Wei-wei, "Design and analysis of a broad band wide beam circular polarization microstrip antenna." Proc. Int. Conf. Microw. Millimeter Wave Technol., vol. 3, pp. $1-4,2012$.

[20] D. Fan, Z. Wang, B. Huang, and W. Zhang, "Design of broad beam circular-polarized microstrip antenna." International Symposium on Antenna \& Propagation. Nanjing, vol. 1, pp. 439-441, Oct. 2013.

[21] H. Nguyen-Bich, H. Chu-Ba, and C. Dao-Ngoc, "All GPS bands dielectric resonator antenna with circular polarization for GPS application." ATC, pp. 65-68, 2012.

[22] K. Saurav, D. Sarkar, A. Singh, and K. V. Srivastava, "Multiband Circularly Polarized Cavity-Backed Crossed Dipole Antenna" IEEE Transactions on Antennas and Propagation, vol. 63, no. 10, pp. 42864296, 2015.

[23] J. Cardoso, and A. Safaai-Jazi, "A spherical helical antenna." IEEE, Antennas and Propagation, 1993, AP-S Digest: 1558-1561.

[24] A. Gu, S. Yang, and Z. Nie, "Analysis and design of miniaturized ultrawideband conical log spiral antennas." Proc. Cross Strait Quad-Regional Radio Sci. Wireless Technol. Conf., pp. 191-194, Jul. 2013.

[25] G. H. Brown and O. M. Woodward, "Circularly-polarized omnidirectional antenna," RCA Review, vol. 8, no. 2, pp. 259-269, June 1947.

[26] V.Galindo,K.Green,"A near-isotropic circulary polarized antenna for space vehicles," IEEE Trans. Antennas and Propag.,vol.13,no.6,pp. 872877, Nov.1965.

[27] M. Huchard, C. Delaveaud, and S. Tedjini, "Miniature antenna for circularly polarized quasi isotropic coverage," The Second European Conference on Antennas and Propagation, EuCAP, pp.1-5, 2007.

[28] H. H. Tran, S. X. Ta, and I. Park, "A compact circularly polarized crosseddipole antenna for an RFID tag," IEEE Antennas and Wireless Propagation Letters, vol. 14, pp. 674-677, 2014.

[29] S. Choi, and K. Sarabandi, "A W-Shaped Antenna With Spatial Polarization Variation for Direction Finding," IEEE Antennas and Wireless Propagation Letters, vol. 17, no. 12, pp. 2429-2433, 2018.

[30] D. S. Bugnolo, "A quasi-isotropic antenna in the microwave spectrum," IRE Trans. Antennas Propag., vol. 10, no. 4, pp. 377-383, Jul 1962.

[31] K.Klionovski, M. B. Arboleda, Z. Su and A. Shamim, "On Synthesis of Orientation Insensitive Antennas", Antennas and Propog. 2019. IEEE Indian Conference on, (InCAP), in proc.

[32] C. Balanis, Antenna Theory: Analysis and Design, 4rd Edition. Hoboken, NJ, USA: Wiley, 2016.

[33] Z. Su, K. Klionovski, H. Liao, Y. Chen, A. Elsherbeni, and A. Shamim, "3D Antenna in Package Design: Maximizing Radiation Pattern 
Isotropicity and CP Coverage," IEEE Int. Symp. Antennas Propag. USNC/URSI Nat. Radio Sci. Meeting, in Proc.

[34] Wang, D., Tan, D. and Liu, L. "Particle swarm optimization algorithm: an overview". Soft Comput No. 22, 387-408 (2018). https://doi.org/10. 1007/s00500-016-2474-6

[35] A. F. Morabito, L. Di Donato, and T. Isernia, "Orbital angular momentum antennas: Understanding actual possibilities through the aperture antennas theory," IEEE Antennas and Propagation Magazine, vol. 60, n. 2, pp. 5967, 2018 